\title{
COCYCLES AND LOCAL PRODUCT DECOMPOSITION
}

BY

\author{
JUN-ICHI TANAKA ${ }^{1}$
}

Dedicated to Professor Haruo Sunouchi on his 60 th birthday

\begin{abstract}
As an application of cocycles, we establish a relation between the classical Hardy spaces on the real line $R$ and simply invariant subspaces on a quotient of the Bohr group. When this result is specialized suitably, it yields the well-known results concerning the elements of invariant subspaces. We also study, by using Gamelin's representation theorem, unitary functions which are the values of cocycles.
\end{abstract}

1. Preliminaries. Let $K$ be a compact abelian group, not a circle, dual to a subgroup $\Gamma$ of the discrete real line $R_{d}$. For each $t$ in $R, e_{t}$ is the element of $K$ defined by $e_{t}(\lambda)=e^{i \lambda t}$ for all $\lambda$ in $\Gamma$. Choose and fix a positive $\gamma$ in $\Gamma$, and let $K_{\gamma}$ be the compact subgroup consisting of all $x$ in $K$ such that $x(\gamma)=1$. Then $K$ may be identified measure-theoretically, and almost topologically, with $K_{\gamma} \times[0,2 \pi / \gamma)$ via the mapping $y+e_{s}$ to $(y, s)$. We suppose for simplicity that $2 \pi$ lies in $\Gamma$ in this section, and $\S \S 2$ and 4 . Thus $K$ may be regarded as $K_{2 \pi} \times[0,1)$. Let $\sigma$ and $\sigma_{1}$ be the normalized Haar measures on $K$ and $K_{2 \pi}$, respectively. Then we may consider $d \sigma=d \sigma_{1} \times d t$ on $K_{2 \pi} \times[0,1)$.

Our objective in this note, by using this local product decomposition, is to show the fact that a certain class of analytic functions on $K_{2 \pi} \times R$ has a close connection to simply invariant subspaces on $K$. In the next section, our characterization of simply invariant subspaces, Theorem 2.1 , is obtained. In $\S 3$ we investigate the values of cocycles and answer a question of Helson. We close with some remarks in $\S 4$.

For any simply invariant subspace $\Re$ of $L^{2}(\sigma)$, we define

$$
(\Re)_{+}=\bigcap_{\lambda<0} \chi_{\lambda} \Re \text { and }(\Re)_{-}=\text {the closure } \bigcup_{\lambda>0} \chi_{\lambda} \Re,
$$

where $\chi_{\lambda}$ denotes the character on $K$ determined by $\lambda$ in $\Gamma$. Then $\Re$ is called to be normalized if $\Re=(\Re)_{+}$. Complex-valued functions of modulus one are said to be unitary functions. A cocycle is a unitary Borel function $A(x, t)$ on $K \times R$ which satisfies the cocycle identity

$$
A(x, t+u)=A(x, t) A\left(x+e_{t}, u\right)
$$

Received by the editors June 4, 1982.

1980 Mathematics Subject Classification. Primary 43A17, 46J15; Secondary 30H05.

Key words and phrases. Cocycles, simply invariant subspaces, compact groups with ordered duals.

${ }^{1}$ This research was partially supported by a Grant-in-Aid for Scientific Research (No. 57740097) from the Japanese Ministry of Education. 
for all $x$ in $K$ and $s, t, u$ in $R$. A cocycle is trivial (resp. a coboundary) if it has the form $e^{i r t} p(x) \overline{p\left(x+e_{t}\right)}$ (resp. $p(x) \overline{p\left(x+e_{t}\right)}$ ) for some $r$ in $R$ and some unitary function $p$ on $K$. There exists a one-to-one correspondence between normalized simply invariant subspaces and cocycles [4, Chapter 2].

We denote by $H^{p}(\sigma)$ and $H^{p}(d t), 0<p \leqslant \infty$, the usual Hardy spaces on $K$ and $R$, respectively. It is known that $H^{1}(d t)$ is the space of all functions in $L^{1}(d t)$ whose Fourier transforms vanish on the negative real line. We let $H^{\infty}\left(d t /\left(1+t^{2}\right)\right)=$ $H^{\infty}(d t)$, that is, the space of all the boundary functions of bounded analytic functions in the upper half-plane. The closure of $H^{\infty}\left(d t /\left(1+t^{2}\right)\right)$ in $L^{p}\left(d t /\left(1+t^{2}\right)\right)$ is denoted by $H^{p}\left(d t /\left(1+t^{2}\right)\right), 0<p<\infty$. Recall that the class of continuous function $\phi$ in $H^{p}(d t)$ with $|\phi(t)|=O\left(t^{-2}\right)$ (as $\left.|t| \rightarrow \infty\right)$ is dense in $H^{p}(d t), 0<p<$ $\infty$ (cf. [3, Chapter II, §3]). Also recall that $\phi$ lies in $H^{p}\left(d t /\left(1+t^{2}\right)\right)$ if and only if $\phi(t)(t+i)^{-2 / p}$ lies in $H^{p}(d t)$.

We refer the reader to [4 and 2, Chapter VII] for further details of analyticity on compact abelian groups and to [1 and 3] for results about classical Hardy spaces.

The following lemma is a minor variation of [4, Theorem 17] so the proof will be omitted.

LEMMA 1.1. Let $\Re_{A}$ be the normalized simply invariant subspace of $L^{2}(\sigma)$ associated with a cocycle $A$. Then for any $f$ in $L^{\infty}(\sigma)$, the following are equivalent:

(i) flies in $\Re_{A}$;

(ii) the function of $t, A(y, t) f\left(y+e_{t}\right)$, lies in $H^{\infty}\left(d t /\left(1+t^{2}\right)\right)$ for $\sigma_{1}$-a.a. $y$ in $K_{2 \pi} ;$ and

(iii) the function of $t, \overline{A(y, t)} \overline{f\left(y+e_{t}\right)}$, is orthogonal to $H^{1}(d t)$ for $\sigma_{1}-a . a . y$ in $K_{2 \pi}$.

We next consider certain spaces of analytic functions on $K_{2 \pi} \times R$. Let $\mathcal{H}$ be the space of all bounded Borel functions $f(y, t)$ on $K_{2 \pi} \times R$ which satisfy

(1.2) the function of $t, f(y, t)$, belongs to $H^{1}(d t)$ for $\sigma_{1}$-a.a. $y$ in $K_{2 \pi}$, and

(1.3) ess.sup $\left\{|f(y, t)| ;(y, t)\right.$ in $\left.K_{2 \pi} \times[n, n+1)\right\}=O\left(n^{-2}\right)$.

We denote by $\mathcal{H}^{p}, 0<p<\infty$, the closure of $\mathcal{H} \cap L^{p}\left(d \sigma_{1} \times d t\right)$ in $L^{p}\left(d \sigma_{1} \times d t\right)$, where we use the ordinary metric on $L^{p}\left(d \sigma_{1} \times d t\right)$ when $0<p<1$. Let $B(y, t)$ be a unitary function on $K_{2 \pi} \times R$. Then for each $f$ in $\mathcal{K}$, we define a bounded Borel function $\Phi_{B}(f)$ on $K$ by

$$
\Phi_{B}(f)(y, s)=\sum_{n=-\infty}^{\infty} \overline{B\left(y-e_{n}, s+n\right)} f\left(y-e_{n}, s+n\right)
$$

for each $(y, s)$ in $K_{2 \pi} \times[0,1)$. Then $\Phi_{B}$ is a linear mapping of $\mathcal{H}$ into $L^{\infty}(\sigma)$. Moreover, for any $p, 0<p \leqslant 1$, it can be easily seen that the restriction of $\Phi_{B}$ to $\mathcal{H} \cap L^{p}\left(d \sigma_{1} \times d t\right)$ may be extended to a bounded linear mapping of $\mathcal{H}^{p}$ into $L^{p}(\sigma)$ (cf. [7, Lemma 1]).

2. Cocycles and the space $\mathcal{H}$. We may now state our main result.

THEOREM 2.1. Let $\mathfrak{N}_{A}$ be the simply invariant subspace of $L^{2}(\sigma)$ associated with a cocycle $A$. Then $\Phi_{A}(\mathcal{H})$ is dense in $\left(\mathfrak{N}_{A}\right)_{-}$. 
Proof. We first note that, for each $y=(y, 0)$ in $K_{2 \pi} \times[0,1), y+e_{t}=(y, 0)+e_{t}$ $=\left(y+e_{[t]}, t-[t]\right)$, where $[t]$ is the largest integer not exceeding $t$. It follows from the cocycle identity (1.1) that

$$
\begin{aligned}
A(y, t) \overline{A\left(y-e_{n-[t]}, n+t-[t]\right)} & =A(y, t) \overline{A\left(y-e_{n-[t]}, n-[t]\right)} \overline{A(y, t)} \\
& =\overline{A\left(y-e_{n-[t]}, n-[t]\right)}
\end{aligned}
$$

for each $(y, t)$ in $K_{2 \pi} \times R$. Hence if $f(y, t)$ lies in $\mathcal{H}$, then we obtain

$$
\begin{aligned}
A(y, t) \Phi_{A}(f)\left(y+e_{t}\right) & =A(y, t) \Phi_{A}(f)\left(y+e_{[t]}, t-[t]\right) \\
& =\sum_{n=-\infty}^{\infty} \overline{A\left(y-e_{n-[t]}, n-[t]\right)} f\left(y-e_{n-[t]}, n+t-[t]\right)
\end{aligned}
$$

by (1.4). Let $\phi$ be any function in $H^{1}(d t)$. Then the property (1.3) assures that

$$
\begin{aligned}
\int_{-\infty}^{\infty} A & (y, t) \Phi_{A}(f)\left(y+e_{t}\right) \phi(t) d t \\
& =\sum_{n=-\infty}^{\infty} \sum_{k=-\infty}^{\infty} \int_{k}^{k+1} \overline{A\left(y-e_{n-[t]}, n-[t]\right)} f\left(y-e_{n-[t]}, n+t-[t]\right) \phi(t) d t \\
& =\sum_{n=-\infty}^{\infty} \sum_{k=-\infty}^{\infty} \int_{k}^{k+1} \overline{A\left(y-e_{n-k}, n-k\right)} f\left(y-e_{n-k}, t+n-k\right) \phi(t) d t \\
& =\sum_{m=-\infty}^{\infty} \sum_{n-k=m} \int_{k}^{k+1} \overline{A\left(y-e_{n-k}, n-k\right)} f\left(y-e_{n-k}, t+n-k\right) \phi(t) d t \\
& =\sum_{m=-\infty}^{\infty} \sum_{k=-\infty}^{\infty} \int_{k}^{k+1} \overline{A\left(y-e_{m}, m\right)} f\left(y-e_{m}, t+m\right) \phi(t) d t \\
& =\sum_{m=-\infty}^{\infty} \overline{A\left(y-e_{m}, m\right)} \int_{-\infty}^{\infty} f\left(y-e_{m}, t+m\right) \phi(t) d t=0
\end{aligned}
$$

for $\sigma_{1}$-a.a. $y$ in $K_{2 \pi}$. Therefore, by Lemma 1.1, $\Phi_{A}(f)$ belongs to $\Re_{A}$. Let $p$ be a function in $\mathscr{N}_{A}$ which is orthogonal to $\Phi_{A}(\mathcal{H})$. We set $g(y, t)=p\left(y+e_{t}\right)$. Then it can be seen that

$$
\int_{K} \overline{p(x)} \Phi_{A}(f)(x) d \sigma(x)=\iint_{K_{2 \pi} \times R} \overline{g(y, t)} \overline{A(y, t)} f(y, t) d \sigma_{1}(y) \times d t=0
$$

for each $f$ in $\mathcal{H}$. This implies that the function of $t, \overline{A(y, t)} \overline{p\left(y+e_{t}\right)}$, lies in $H^{2}\left(d t /\left(1+t^{2}\right)\right)$ for $\sigma_{1}$-a.a. $y$ in $K_{2 \pi}$. On the other hand, since the function of $t$, $A(y, t) p\left(y+e_{t}\right)$, lies in $H^{2}\left(d t /\left(1+t^{2}\right)\right)$, it must be constant. Hence $|p|$ is constant on $K$. From this, we may assume that $p$ is a unitary function on $K$. Thus we have $A(x, t)=p(x) \overline{p\left(x+e_{t}\right)}$ and $\Re_{A}=p H^{2}(\sigma)$. This completes the proof.

We collect some corollaries following from Theorem 2.1. Recall that a cocycle $A(x, t)$ is continuous if $A(y, t)$ is continuous on $K_{2 \pi} \times R$ as a function of $(y, t)$ [4, Chapter 5]. Let $C_{0}\left(K_{2 \pi} \times R\right)$ denote the space of all continuous functions on $K_{2 \pi} \times R$ which vanish at infinity. We notice that $\mathcal{H} \cap C_{0}\left(K_{2} \times R\right)$ is dense in $\mathcal{H}$ as 
a subspace of $L^{1}\left(d \sigma_{1} \times d t\right)$, and that $\Phi_{A}(f)$ lies in $C(K)$ for any $f$ in

$$
\mathcal{H} \cap C_{0}\left(K_{2 \pi} \times R\right) \text {. }
$$

These facts easily imply the following.

Corollary 2.2. Let $A$ and $\mathfrak{R}_{A}$ be as in Theorem 2.1. If $A$ is continuous, then $\left(\Re_{A}\right)_{-} \cap C(K)$ is dense in $\left(\Re_{A}\right)_{-}$.

We next give another proof of Helson's existence theorem $[4$, Theorem 16; 8, 10].

Corollary 2.3. Let $A$ and $\Re_{A}$ be as in Theorem 2.1. Then $\Re_{A}$ contains a unitary function.

Proof. Define a function $w(t)$ in $L^{1}(d t)$ by

$$
w(t)= \begin{cases}n^{-2} & \text { on }[n, n+1),|n| \geqslant 1, \\ 3 \sum_{j=1}^{\infty} j^{-2} & \text { on }[0,1) .\end{cases}
$$

It is easy to see that $\log w(t)$ belongs to $L^{1}\left(d t /\left(1+t^{2}\right)\right)$. Hence there is a function $\phi$ in $H^{1}(d t)$ such that $|\phi(t)|=w(t)$ (cf. [3, Chapter II, Theorem 4.4]). If we set $f(y, t)=\phi(t)$, then $\Phi_{A}(f)$ lies in $\Re_{A}$ and $\left|\Phi_{A}(f)\right| \geqslant \sum_{j=1}^{\infty} j^{-2}$ on $K$. Thus it follows from Szegö's theorem that $\mathfrak{K}_{A}$ contains a unitary function.

COROLlaRY 2.4. Let $A$ and $\Re_{A}$ be as in Theorem 2.1. Then there exists a function $f$ in $\Re_{A}$ satisyfing:

(i) the function of $t, f\left(y+e_{t}\right)$, can be extended analytically to $\{z ; \operatorname{Im} z>-\sqrt{3} / 2\}$ for each $\sigma_{1}$-a.a. $y$ in $K_{2 \pi}$;

(ii) $\log |f(x)|$ belongs to $L^{1}(\sigma)$; and

(iii) for any positive $\lambda$ in $\Gamma, f$ does not lie in $\chi_{\lambda} \Re_{A}$.

Proof. Let $h(y, t)=4 /(2 t-1+\sqrt{3} i)^{2}$. Then $|h(y, t)| \geqslant 1$ on $K_{2 \pi} \times[0,1]$, and $|h(y, t)|<1$ otherwise. So by Theorem 2.1 we may choose an integer $m$ such that the function $g_{1}=\Phi_{A}\left(h^{m}\right)$ in $\Re_{A}$ satisfies (i) and $\left|g_{1}(y, s)\right|>1$ on $K_{2 \pi} \times\left[\frac{1}{6}, \frac{5}{6}\right)$. It may be assumed that $g_{1}$ has property (iii). Similarly, we can construct a function $g_{2}$ in $\Re_{A}$ which satisfies (i) and $\left|g_{2}(y, s)\right|>1$ on $K_{2 \pi} \times\left\{\left[0, \frac{2}{6}\right) \cup\left(\frac{4}{6}, 1\right)\right\}$. It follows from Jensen's inequality and Fubini's theorem that

$$
\begin{aligned}
\frac{1}{2 \pi} \int_{K} \int_{0}^{2 \pi} \log \mid & g_{1}(x)+e^{i \theta} g_{2}(x) \mid d \sigma(x) d \theta \\
& \geqslant \int_{K} \max \left(\log \left|g_{1}(x)\right|, \log \left|g_{2}(x)\right|\right) d \sigma(x) \geqslant 0 .
\end{aligned}
$$

Thus there exists a $\theta$ in $[0,2 \pi)$ for which $f=g_{1}+e^{i \theta} g_{2}$ has the desired properties.

The above corollary is the main step in the proof of [4, Theorem 26].

We may easily choose a unitary function $B$ on $K_{2 \pi} \times R$ such that the closure [ $\left.\Phi_{B}(\mathcal{H})\right]_{2}$ of $\Phi_{B}(\mathcal{H})$ in $L^{2}(\sigma)$ is a doubly invariant subspace, so it is worthwhile to note a condition under which $\left[\Phi_{B}(\mathcal{H})\right]_{2}$ is simply invariant. 
THEOREM 2.5. Let $B$ be a unitary function on $K_{2 \pi} \times R$. Then $\left[\Phi_{B}(\mathcal{H})\right]_{2}$ is simply invariant if and only if there exists a cocycle $A(x, t)$ for which

(2.1) the function of $t, A(y, t) \overline{B(y, t)}$, belongs to $H^{\infty}\left(d t /\left(1+t^{2}\right)\right)$ for $\sigma_{1}-a . a . y$ in $K_{2 \pi}$.

Proof. Suppose that there exists a cocycle $A$ with property (2.1). Then $A \bar{B} f$ lies in $\mathcal{H}$ for each $f$ in $\mathcal{H}$. Since $\Phi_{B}(f)=\Phi_{A}(A \bar{B} f)$, it follows from Theorem 2.1 that $\left[\Phi_{B}(\mathcal{H})\right]_{2}$ is a simply invariant subspace. Conversely, suppose that $\left[\Phi_{B}(\mathcal{H})\right]_{2}$ is simply invariant, and let $A$ be the cocycle of $\left(\left[\Phi_{B}(\mathcal{H})\right]_{2}\right)_{+}$. Let $\phi h$ be a function in $\mathcal{H}$ which is the product of a function $\phi$ in $H^{1}(d t)$ times a function $h$ in $C\left(K_{2 \pi}\right)$. We notice that

$$
A(y, t)=A(y,[t]-n) A\left(y+e_{[t]-n}, t-[t]+n\right)
$$

and

$$
\begin{aligned}
\Phi_{B}(\phi h)\left(y+e_{t}\right) & =\Phi_{B}(\phi h)\left(y+e_{[t]}, t-[t]\right) \\
& =\sum_{n=-\infty}^{\infty} \overline{B\left(y+e_{[t]-n}, t-[t]+n\right)} \phi(t-[t]+n) h\left(y+e_{[t]-n}\right) .
\end{aligned}
$$

It follows from Lemma 1.1 and an argument similar to the proof of Theorem 2.1 that

$$
\begin{aligned}
\int_{-\infty}^{\infty} A(y, t) \Phi_{B}(\phi h)\left(y+e_{t}\right) \psi(t) d t \\
=\sum_{m=-\infty}^{\infty} A(y,-m) h\left(y-e_{m}\right) \\
\quad \times \int_{-\infty}^{\infty} A\left(y-e_{m}, t+m\right) \overline{B\left(y-e_{m}, t+m\right)} \phi(t+m) \psi(t) d t \\
=0
\end{aligned}
$$

for each $\psi$ in $H^{1}(d t)$. Since $h$ is arbitrary in $C\left(K_{2 \pi}\right)$, we have

$$
\int_{-\infty}^{\infty} A\left(y-e_{m}, t+m\right) \overline{B\left(y-e_{m}, t+m\right)} \phi(t+m) \psi(t) d t=0
$$

for each integer $m$. This implies that $A$ satisfies (2.1).

3. Cocycles and unitary functions. Let $A(x, t)$ be a cocycle on $K$. In [5, §4], Helson has shown that if the function of $x, A_{u}(x)=A(x, u)$, lies in $H^{2}(\sigma)$, then it must be constant. This odd result grew out of a basic problem concerning spectrum of cocycles. We provide, by using Gamelin's representation theorem, some remarks on this theorem.

In this section we do not assume $2 \pi$ belongs to $\Gamma$. Let $K_{\gamma}$ be as in $\S 1$ for a positive $\gamma$ in $\Gamma$, and put $u=2 \pi / \gamma$. We denote by $\mathcal{U}\left(K_{\gamma}\right)$ and $\mathcal{Q}(K)$ the classes of all unitary functions on $K_{\gamma}$ and $K$, respectively. We first recall the definition of cocycles introduced by Gamelin (see [2, Chapter VII, $\$ 11])$. For any $\beta$ in $\mathcal{Q}\left(K_{\gamma}\right)$ the cocycle 
$B_{\beta}(x, t)$ is given explicitly for positive $t$ by

$$
B_{\beta}(y, t)= \begin{cases}1 & \text { on } K_{\gamma} \times[0, u), \\ \prod_{j=0}^{m-1} \beta\left(y+e_{j u}\right) & \text { on } K_{\gamma} \times[m u,(m+1) u)\end{cases}
$$

for each positive integer $m$, and $B_{\beta}\left(y+e_{s}, t\right)=B_{\beta}(y, s+t)$ for $s$ in $[0, u)$. Then $B_{\beta}$ is trivial if and only if there is an $f$ in $\mathcal{Q}\left(K_{\gamma}\right)$ and some $r$ in $R$ for which $\beta$ can be expressed in the form $\beta(y)=e^{i r} f(y) \overline{f\left(y+e_{u}\right)}$ for a.a. $y$ in $K_{\gamma}[4$, Chapter $4, \S 9]$. Gamelin's representation theorem [2, Chapter VII, Theorem 11.1] asserts that every cocycle $A$ on $K$ has the factorization $A=B_{\beta} C$, where $\beta$ is a function in $\mathcal{Q}\left(K_{\gamma}\right)$, and $C$ is a coboundary.

The following theorem shows vaguely which unitary functions on $K$ are the values of cocycles and settles a question posed by Helson [5, $\$ 1]$ : Is the class of all $A_{1}$ on $K$ different from the class of all $A_{2}$ ?

TheOrem 3.1. For any positive $u$ in $R$, let $\gamma=2 \pi / u$, and let $\left\{A_{u}\right\}$ denote the class of all the values $A_{u}(x)=A(x, u)$ of cocycles $A$. Then we obtain the following properties:

(i) if $\gamma$ belongs to $\Gamma$, then every $A_{u}$ in $\left\{A_{u}\right\}$ has the form

$$
A_{u}(x)=\beta(y) q(x) \overline{q\left(x+e_{u}\right)}
$$

for $\sigma$-a.a. $x=(y, s)$ in $K_{\gamma} \times[0, u)$, where $\beta$ is a function in $\mathscr{Q}\left(K_{\gamma}\right)$ and $q$ is a function in $\mathrm{Q}(K)$;

(ii) for each positive integer $m,\left\{A_{u}\right\}$ contains $\left\{A_{m u}\right\}$; and

(iii) if $\gamma$ belongs to $\Gamma$, then for any $v$ in $(0, u)$, there exists an $A_{v}$ in $\left\{A_{v}\right\}$ which does not lie in $\left\{A_{u}\right\}$.

Proof. (i) is a direct consequence of Gamelin's representation theorem, so it is enough to show (ii) and (iii). We notice that if $A(x, t)$ is a cocycle, then so is $A\left(x+x_{0}, t\right)$ for any fixed $x_{0}$ in $K$, and the product of two cocycles is also a cocycle. For any positive integer $m$, we set

$$
B(x, t)=A(x, t) A\left(x+e_{u}, t\right) \cdots A\left(x+e_{(m-1) u}, t\right) .
$$

Then it follows from the cocycle identity (1.1) that $B(x, t)$ is a cocycle which satisfies $B(x, u)=A(x, m u)$. Thus we have (ii). On the other hand, by Gamelin's representation theorem, we may choose a function $\beta$ in $\mathcal{Q}\left(K_{\gamma}\right)$ for which $B_{\beta}$ is a nontrivial cocycle. We now show that for each $v$ in $(0, u), B_{\beta}(x, v)$ cannot belong to $\left\{A_{u}\right\}$. By Definition (3.1), it can be seen that

$$
B_{\beta}(x, v)= \begin{cases}1 & \text { for } x=(y, s) \text { in } K_{\gamma} \times[0, u-v) \\ \beta(y) & \text { for } x=(y, s) \text { in } K_{\gamma} \times[u-v, u)\end{cases}
$$


Suppose to the contrary that $B_{\beta}(x, v)$ belongs to $\left\{A_{u}\right\}$. Then by (i) there are functions $\alpha$ in $\mathscr{Q}\left(K_{\gamma}\right)$ and $p$ in $\mathscr{Q}(K)$ such that

$$
B_{\beta}(x, v)=\alpha(y) p(x) \overline{p\left(x+e_{u}\right)}
$$

for $\sigma$-a.a. $x=(y, s)$ in $K_{\gamma} \times[0, u)$. Therefore it follows from (3.2) and Fubini's theorem that there is an $s$ in $[0, u-v)$ such that $\alpha(y)=\overline{p((y, s))} p\left(\left(y+e_{u}, s\right)\right)$ for a.a. $y$ in $K_{\gamma}$. From this fact, we can easily see that $\beta(y)=\delta(y) \overline{\delta\left(y+e_{u}\right)}$ for some $\delta$ in $\mathcal{Q}\left(K_{\gamma}\right)$, so $B_{\beta}$ must be trivial. Thus we have a contradiction, and this completes the proof.

From (ii) and (iii) of Theorem 3.1 we have

Corollary 3.2. If $\pi$ belongs to $\Gamma$, then $\left\{A_{1}\right\}$ contains strictly $\left\{A_{2}\right\}$.

4. Remarks. We recall that a Borel function $f$ on $K_{2 \pi} \times R$ is automorphic if $f(y, s+1)=f\left(y+e_{1}, s\right)$ a.a. on $K_{2 \pi} \times R$, and any Borel function on $K=K_{2 \pi} \times$ $[0,1)$ can be extended uniquely to be automorphic on $K_{2 \pi} \times R$ [2, Chapter VII, §6].

(a) Let $\tilde{H}^{\infty}(\sigma)$ be the space of all automorphic extensions of functions in $H^{\infty}(\sigma)$. The following question is interesting and probably difficult:

For any cocycle $A$, does there exist an $f$ in $\mathcal{H}^{1}$ for which $\left(f+\Phi_{A}^{-1}(\{0\})\right) \cdot \tilde{H}^{\infty}(\sigma)$ is dense in $\mathcal{H}^{1}$ ?

This is related to the old problem of whether every simply invariant subspace is generated by one of its elements. Indeed, if we could choose such a function $f$, then $\Phi_{A}(f)$ would be a single generator of $\left(\Re_{A}\right)_{-}$.

(b) We know that the dual space of $H^{p}(\sigma), 0<p<1$, has dimension one [7,9]. By the argument of [7], Theorem 2.1 provides an extension of this result:

Let $\Re$ be a simply invariant subspace of $L^{p}(\sigma), 0<p<1$. Then the dual space of Th has at most dimension one.

(c) Let $\Re$ be a simply invariant subspace of $L^{2}(\sigma)$. For any $f$ in $\Re$, let $\tilde{f}$ denote the automorphic extension of $f$ to $K_{2 \pi} \times R$. Then we may easily verify that there exists a unitary function $q$ on $K_{2 \pi} \times R$ such that the closure $[\tilde{f} \mathcal{H}]_{1}$ of $\tilde{f} \mathcal{H}$ in $L^{1}\left(d \sigma_{1} \times d t\right)$ coincides with $q \mathcal{H}^{1}$. Let $p(x)=p(y, s)$ be the restriction of $q$ to $K_{2 \pi} \times[0,1)$, and set $\beta(y, s)=q(y, s+1) \overline{q\left(y+e_{1}, s\right)}$. Since $\tilde{f}$ is automorphic, $\beta$ defines a unitary function on $K_{2 \pi}$. We denote by $C_{\beta}$ the cocycle defined by (3.1). Then it can be seen that the cocycle $C_{\beta}(x, t) p(x) p\left(x+e_{t}\right)$ corresponds to the simply invariant subspace generated by $f($ cf. $[5, \S 3])$. Similarly, let $\tilde{\mathscr{N}}$ denote the space of all automorphic extensions of functions in $\mathfrak{N}$. Then it is not hard to see that $[\tilde{\mathscr{N}} \cdot \mathcal{H}]_{1}=q \mathcal{H}^{1}$ for some unitary function $q$ on $K_{2 \pi} \times R$. Thus in the same manner, we may find the cocycle associated with $\Re$. This provides another naive definition of cocycles (cf. [4, Chapter 2]).

\section{REFERENCES}

1. P. Duren, Theory of $H^{p}$ spaces, Academic Press, New York, 1970.

2. T. Gamelin, Uniform algebras, Prentice-Hall, Englewood Cliffs, N. J., 1969.

3. J. Garnett, Bounded analytic functions, Academic Press, New York, 1981.

4. H. Helson, Analyticity on compact abelian groups, Algebras in Analysis, Academic Press, New York, 1975, pp. $1-62$. 
5. __ Compact groups with ordered duals. VI, Bull. London Math. Soc. 8 (1976), 278-281.

6. P. Muhly, Function algebras and flows, Acta Sci. Math. (Szeged) 35 (1973), 111-121.

7. Ergodic Hardy spaces and duality, Michigan Math. J. 25 (1978), 317-323.

8. T. Nakazi, Helson's existence theorem of function algebras, Arch. Math. 32 (1979), 385-390.

9. J. Shapiro, Subspaces of $L^{p}(G)$ spanned by characters: $0<p<1$, Israel J. Math. 29 (1978), 248-264.

10. J.-I. Tanaka, A note on Helson's existence theorem, Proc. Amer. Math. Soc. 69 (1978), 87-90.

11. Quasi-invariant measures and maximal algebras on minimal flows, Michigan Math. J. 29 (1982), 199-211.

Department of Mathematics, Tsuru University, Tsuru City Yamanashi-Ken, 402 Japan 